\title{
Weitere Beiträge zur Kenntnis anorganischer Kolloide.
}

\author{
Von \\ A. Gutbier und G. Hormeine.
}

Während unserer Untersuchungen über die Kolloide haben wir uns auch bemüht, die Hydrosole des Wismuts, des Kupfers und des Quecksilbers darzustellen und beabsichtigen, im folgenden über diese Versuche kurz zu berichten.

\section{Wismut.}

Bereits in Jahre 1853 hat R. SCHNEIDER ${ }^{1}$ Reduktionsversuche von weinsaurem Wismutoxydkalium mittels Zinnchlorür ausgeführt und mitgeteilt, dals er hierbei eine undurchsichtige, braungefärbte Flüssigkeit erhalten habe, die er für eine Lösung von zinnsaurem Wismutoxydul hielt. Da aber in dieser Flüssigkeit auf Zusatz von Elektrolyten Sedimentation eintritt, so wiesen Vanino und TRECBenT ${ }^{2}$ mit Recht darauf hin, dafs das Wismut höchstwahrscheinlich in kolloidal gelöstem Zustande als Metall in der Flüssigkeit enthalten sein würde; A. LotTERMoser ${ }^{3}$ gelangte denn auch $\mathrm{zu}$ dem Wismutsol, als er eine schwach salpetersaure Wismutnitratlösung bei Gegenwart von Ammoniumzitrat und Ammoniak mittels Zinnchlorür reduzierte.

Bei unseren Arbeiten über die reduzierende Wirkung von Hydrazinhydrat haben wir auch Versuche angestellt, das flüssige Fydrosol des Wismuts mit Hilfe des genannten Reduktionsmittels darzustellen, doch hat es sich gezeigt, dals sich gerade in diesem Falle Hydrazinhydrat absolut reaktionsunfähig zeigt.

\footnotetext{
1 Pogg. Ann. 88, 45.

${ }^{2}$ Ber. deutsch. chem. Ges. 32, 1072.

${ }^{3}$ Anorganische Kolloide, Stuttgart 1901.

Z. anorg. Chem, Bd. 44 .
} 
Dagegen haben wir in der unterphosphorigen Săure, die ja bekanntlich schon von W. MU'shmass und F. Mawnow ${ }^{1}$ zur quantitativen Bestimmung des Wimuts verwendet worden ist, ein Mittel gefunden, welches die vorübergehende Isolierung von kolloidalem Wismut gestattet.

Versetzt man nămlicheine auf $70-80^{\circ}$ erwärmte sebr verdünnte und nur schwache salzsaure Lösung von Wismutoxychlorid mit einigen Tropfen ca. $10 \%$ iger unterphosphoriger Săure, so trübt sich die Flussigkeit nach einiger Zeit, nimmt eine schmutziggraue Farbe an und scheidet nach nicht zu langer Zeit metallisches Wismut àb.

Trotz der gröfsten Verdünnung und trotz aller Vorsichtsmalsregeln gelang es uns aber doch nicht, llaltbare Lösungen von kolloidalem Wismut zu erhalten; setzt man aber der Wismutchloridlösung vor der Reduktion wieder das gleiche Volumen einer $1 \%$ igen Gummilösung $z a$, so gestaltet sich das Resultat des Versuches ganz anders; denn dann färbt sie die Lösung erst nach längerer Zeit hellbraun und später dunkelbraun, während si $\theta$ im auffallenden Lichte wiederum den bei so vielen Metallsoler. bemerkten Petroleumschimmer zeigt.

Da aber merkwürdigerweise dieser Versuch nicht immer glatt gelingt, haben wir mit folgender geringen Abänderung der Versuchsbedingungen und dann immer mit Erfolg gearbeitet: Eine bestimmte Menge starkverdünnter Gummilösung wurde mit einigen Tropfen unterphosphoriger Säure versetzt, dann gut erwärmt und hierauf mit dem gleichen Volumen einer Wismutchloridlösung vermischt. Nach kurzer Zeit schon färbte sich die Flüssigkeit tiefbraun und zeigte nun, solange sie noch heifs war, alle Eigenschaften einer kolloidalen Lösung: Durch Schütteln mit Baryumsulfat oder mit Tierkohle wurde das Hydrosol zerstört; durch Eindampfen liefs sich die kolloidale Lösung ziemlich stark konzentrieren, ohne Zersetzung zu erleiden.

Bei dem Erkalten der Flüssigkeit machten wir aber folgende interessante Beobachtung: Die Flüssigkeit, welche, wie gesagt, in der Hitze tiefbraun gefärbt war, verlor mit abnehmender Temperatur immer mehr von ihrer Färbung und wurde mit der Zeit wasserklar; derselbe Vorgang trat auch während der Dialyse ein. Wurde nun die Flüssigkeit wieder erwärmt, so entstand - auch bei den dialysierten Pseudolösungen - wieder das dunkelbraun gefärbte, im

I. anorg. Chem. 13, 209. 
auffallenden Lichte Petroleumschimmer zeigende Wismutsol, das dann wkkrend des Exkaltens nach und nach wieder wasserklar wrurde:

Welche Verhältnisse diese interessante Erscheinung hervorrufen, konnten wir leider nicht mit vollständiger Sicherbeit ergrinden; es scheint aber, dafs das kolloidale und somit in feinster Verteilung vorhandeng Wismut an der Luft leicht oxydiert und dann von der vorhandenen Salzsäure wieder aufgelöst wird und dals sich die unterphosphorige Säure, sowie die Salzsäure durch die Dialyse nur schwer entfernen lassen, so dafs beim Erwărmen der Flüssigkeit wieder Reduktion und somit Bildung des Hydrosols stattfindet.

\section{Kupfer.}

In dem Umstande, dals auch dieses Element in fein verteiltem Zustande aufsererdentlich oxydationsfähig ist, ist die allgemein bekannte Schwierigkeit, dieses Metall in dauerhafte. Hydrosole überzuführen, begrtindet.

Bisher ist kolloidales Kupfer nur einmal in reinem Zustande und zwar von J. Brlitzer ${ }^{1}$ gewonnen worden, während A. LorterkMOsER ${ }^{2}$ nur ein mit Zinnsäure gemengtes Präparat erhielt.

Auf Grund der Versuche, welche der eine von uns bereits mitgeteilt hat $^{8}$, haben wir - neben anderen Reduktionsmitteln ${ }^{4}-$ das Hydrazinhydrat verwendet und folgende Resultate erhalten.

Lälst man eine verdunnte Lösung von Hydrazinhydrat $1: 2000$ - auf ammoniakalische Kupfersulfatlösung - $1: 1000-$ in der Wärme einwirken, so erhält man ein Hydrosol, das bei anhaltendem Erhitzen metallisches Kupfer abscheidet.

Bei Gegenwart von Gummiarabikum kann man nun auch wieder dieses Sol in besser haltbarem Zustande gewinnen; eine derartige Flüssigkeit, welche sofort nach der Bereitung in einen vorher längere Zeit in Wasser gelegenen - Pergamentpapiersack eingefullt und vier Tage lang gegen Wasser dialysiert worden war, besafs im auffallenden Lichte eine kupferbraunrote Farbe und erschien im durchfallenden Lichte prachtvoll blau gefärbt.

1 Ber. deutsch. chem. Ges. 35, 1929.

1. c.

A. Gutrier, Z. anorg. Chem. 32, 355.

- Vergl. G. Hormminr, "Über anorganische Kolloide und Kryptoide". Inauguraldissertation, Erlangen 1904. 
Auch dieser Fltzsigkeit kamen alle bekannten Eigenschaften der Hydrosole zu. Unter Abschlufs der Luft hält sich das kolloidale Kupfer ganz gut eine Zeitlang; bleibt es aber der Einwirkung der Luft ausgesetzt, so tritt infolge der leichten Oxydierbarkeit mit der Zeit ein Farbenumschlag ein: die Flhssigkeit verliert zunächst den charakteristischen rotbraunen Schimmer und erscheint schliefslich als eine klare grüne, miturter anch grungelb gefürbte Lösung.

\section{Qneakailber.}

Versuche, das Quecksilber in den kolloidalen Zustand uberzuführen, sind zwar ziemlich häufig aber meist nur ohne Erfolg angestellt worden; nur J. BruLITzER ${ }^{1}$ ist es bisher gelungen, kolloidales Quecksilber in reinem Zustaude zu gewinnen.

Wir haben nun die von dem einen von uns bereits in wässeriger Lösung ausgeführten Reduktionsversuche ${ }^{2}$ unter Anwendung von Quecksilberoxydulnitrat und Quecksilberchloridlösungen bei Gegenwart von Gummiarabikum wiederholt ind vortibergehend das fiüssige Hydrosol des Quecksilbers in Gestalt einer braungefärbten Flüssighreit erbalten, die aber merkwürdigerweise trotz des schützenden Kolloids nur wenig Beständigkeit besafs and schon bald nach der Bereitung eine graue Farbe annahm, um kurz darauf zu koagulieren.

Ebenso ergebnislos verlief auch der Versuch, eine starkverdỉnnte wässerige Lösung von Nwsšsters Reagens bei Gegenwart von Gummiarabikum durch Hydrazinhydrat zn reduzieren; obwohl das flüssige Hydrosol des Quecksilber hierbei gebildet wurde, konnten beständige Lösungen nicht gewonnen werden.

Wir haben daher weitere Versuche in dieser Richtung aufgeben müssen.

11.6.

*. Guthise, 1. c.

s. c. vergl. G. Hormeter, l. c.

Erlangen, Chem. Laboratorium der Kgl. Universität, Dexember 1904.

Bei der Redaktion eingegangen am 11. Februar 1905. 